\title{
Identification of landmarks on lower limb joint from CT images for kinematics studies: a totally semi-automatic procedure
}

\author{
M. Giorgi ${ }^{1}$, B. Innocenti ${ }^{2}$, L. Labey ${ }^{2}$, A. Audenino ${ }^{1} \&$ C. Bignardi $^{1}$ \\ ${ }^{1}$ Department of Mechanics, Politecnico di Torino, Italy \\ ${ }^{2}$ European Centre for Knee Research, Smith \& Nephew, Belgium
}

\begin{abstract}
The identification of an accurate, reliable and patient specific coordinate system for a bone is fundamental to analyzing the kinematics of a human joint. The accuracy in the localization of anatomical landmarks of joint surfaces is extremely important because even a small variation in their positions could induce a high variation in the definition of anatomical axes and further on the kinematics output. The aim of this study was to develop and validate a semiautomatic, accurate, and reproducible routine able to identify the position of anatomical landmarks on joint surfaces. This routine, starting from a CT of a femoral bone, used as input, is able to identify semi-automatically the femoral head and the medial and lateral distal femoral condyles. Moreover, it allows the identification of the following anatomical landmarks: the Femoral Hip Center (FHC), the Femoral Medial Epicondyle (FME) and the Femoral Lateral Epicondyle (FLE). From these points a standard coordinate system of the femur is univocally determined according to previous literature. Compared to other commercial processes, extensively used in this field, one peculiarity of this routine is that it is not necessary to generate a $3 \mathrm{D}$ model of the joint in order to define the anatomical landmarks. Usually, to generate a 3D lower limb model, with the commercial process, 4 to 5 hours are needed, with this approach we can significantly reduce this time. To validate the routine we analyzed ten different CTs of lower limbs. Two different tests were performed. The first test was performed to verify and check the output geometry of the model; the second test was aimed at estimating the repeatability and reproducibility of the procedure. For such a task five different operators identified for each model the three anatomical landmarks, three times each. The Intra-Class Correlation coefficient
\end{abstract}


(ICC) values (intra and inter) obtained for the landmarks were always higher than 0.996. Comparing the results obtained with this routine with the results obtained using largely used commercial software we found a significant reduction of the error as regards the evaluation of landmarks in terms of inter and intra-observer variability. For example, in the worst condition, on the identification of the femoral lateral condyle point (FLE), the same operator found an average and maximum distance between the real point and the landmark found of respectively 3.5 and $8.8 \mathrm{~mm}$ with the use of the commercial software and of respectively 0.8 and $0.9 \mathrm{~mm}$ with the use of our routine.

Keywords: knee joint, landmarks, CT images.

\section{Introduction}

An anatomical landmark is a biologically-meaningful point in an organism. Usually experts define anatomical points to ensure their correspondences within the same species. They provide the link between the CT scan data and surgically relevant reference that can be found by visualization or palpation during the operation. Anatomical landmarks are also important to define anatomical axes. Advances in medical imaging technology have made it possible to routinely acquire high-resolution, 3D images of human anatomy and function using a variety of imaging modalities. The ability to inspect structural relationships in three dimensions and the ever-improving quality of the images have largely increased the number of clinical applications in various medical disciplines, such as neurology, cardiology, surgery, and radiotherapy, that benefit from 3D image information to support critical medical decisions [1]. The use of 3D image processing and visualization techniques allows direct inspection of the scene in three dimensions and facilitates extracting quantitative information. The identification of an accurate, reliable and patient specific coordinate system for a bone [2] and the realization of 3D femoral bone models are fundamental, together with the tibia and patella models, to perform kinematics studies necessary to understand the functioning of an arthroprosthesis.

According to a literature work [3], the aim of this study was to develop a semi-automatic, accurate, reliable and reproducible routine able to generate $3 \mathrm{D}$ models and to identify the position of anatomical landmarks on joint surfaces.

\section{Materials and methods}

Ten unpaired fresh frozen amputated lower limbs were analyzed using a helical CT scan. The specimens were obtained from Caucasian cadavers aged between 78 and 87 years old when they deceased. The images were obtained at $120 \mathrm{kV}$ and $450 \mathrm{~mA}$, with a slice thickness of $1.25 \mathrm{~mm}$ and a pitch of $0.5 \mathrm{~mm} / \mathrm{rev}$.

Starting from the CT of femoral bones, used as input, a semi-automatic routine able to identify the position of anatomical landmarks on joint surfaces was developed. The routine was implemented using MATLAB (MathWorks Natick, Massachusetts, USA) [4] and is divided into four main blocks: 
1. Image enhancement through a semi-automatic procedure based on thresholding technique;

2. generation of nodes, surfaces, volumes constituting the 3D model through a fully automated procedure;

3. viewing and saving the model through a fully automated procedure;

4. identification of anatomical landmarks.

The first block is based on the threshold technique $[5,6]$ and it allows us to prepare all the images for the next step. As is known, the threshold technique is useful to convert images in $\mathrm{B} / \mathrm{W}$ because, given that the values of the pixels that make up the CT images in grayscale are all between 0 and 1 where 0 corresponds to white and 1 corresponds to black, with this technique we can choose a threshold value that establishes which pixels become white and which will become black. If we set a threshold value equal to 0.6 it means that all pixels with values above 0.6 will become 1 so black. In this block the inputs are the original-CT images and, using the threshold technique, we obtain as output the same images converted in $\mathrm{B} / \mathrm{W}$ and cleaned of all the parts that we do not need for our aim, fig. 1.

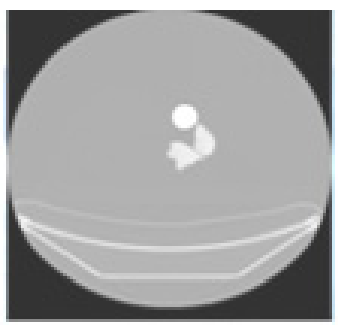

a)

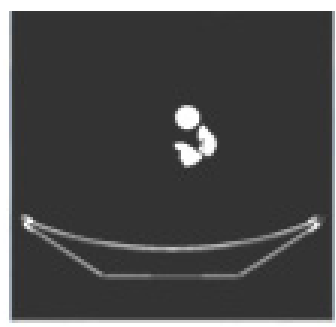

b)

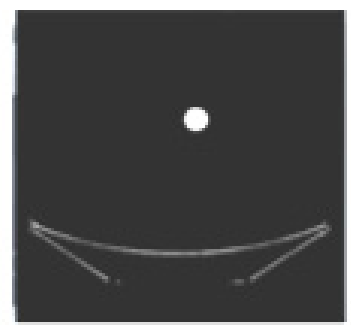

c)

Figure 1: a) original $\mathrm{CT}, \mathrm{b}) 0.72$ threshold value, c) 0.76 threshold value.

The second block generates a volumetric mesh creating the nodes, surfaces and volumes constituting the $3 \mathrm{D}$ model of the bone section under consideration. This procedure is fully automated and is based on a tetrahedral method for the mesh generation [7]. This block takes as input the images prepared on the block before and provides as output all the nodes, elements, and faces constituting the model.

The third block has been implemented to view and save the 3D model; this routine gives the user, two different kinds of visualization; the Mesh Plot and the Point Cloud Plot, illustrated in figure 2. The Mesh Plot function is used to obtain a quick view of the model surface realized to check if there are some anomalies in the model's geometry. The Point Cloud Plot function is used to visualize all the points that constitute the model and for that is a computationally heavy view. 


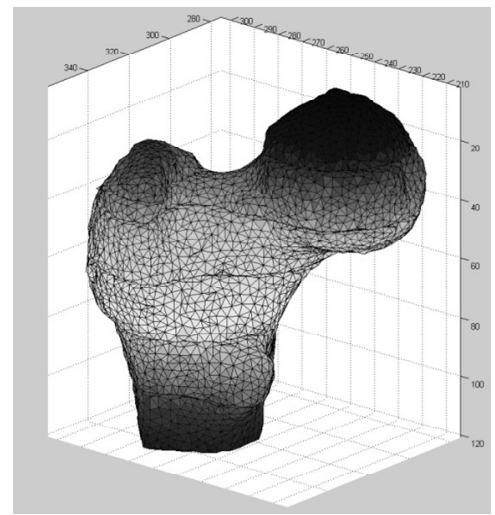

a)

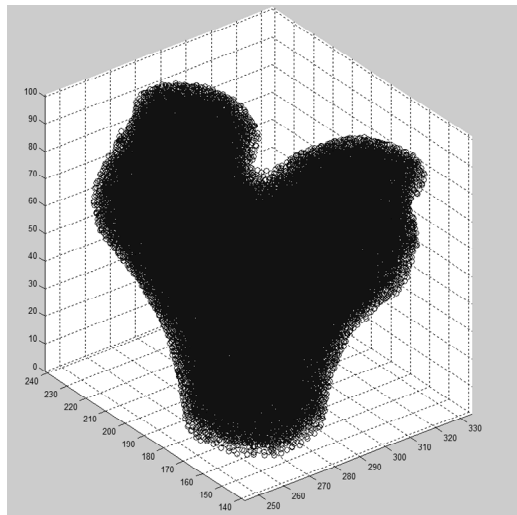

b)

Figure 2: a) Mesh plot, b) point cloud plot.

The anatomical landmarks of the femur identified by the semi-automatic routine are:

- Femoral Hip Center (FHC), defined as the center of best-fit sphere to the head of the femur (fig. 3).

- Femoral Medial Epicondyle (FME), defined as the most anterior and distal osseous prominence over the medial aspect of the medial femoral condyle (fig. 4) [8].

- Femoral Lateral Epicondyle (FLE), defined as the most anterior and distal osseous prominence over the lateral aspect of the lateral femoral condyle (fig. 5) [9].

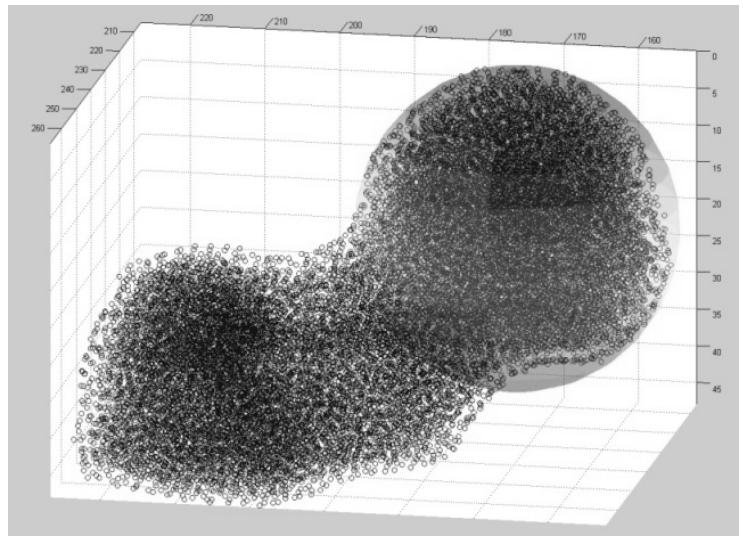

Figure 3: Example of best-fit sphere to the head of the femur.

We chose these anatomical landmarks because, according to literature, from these points a standard coordinate system of the femur is univocally determinate [3]. 


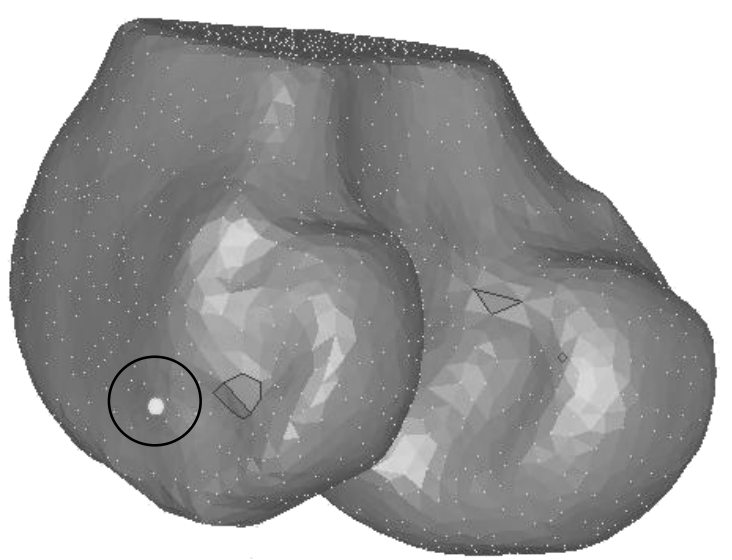

Figure 4: $\quad$ Example of FME found with our routine.

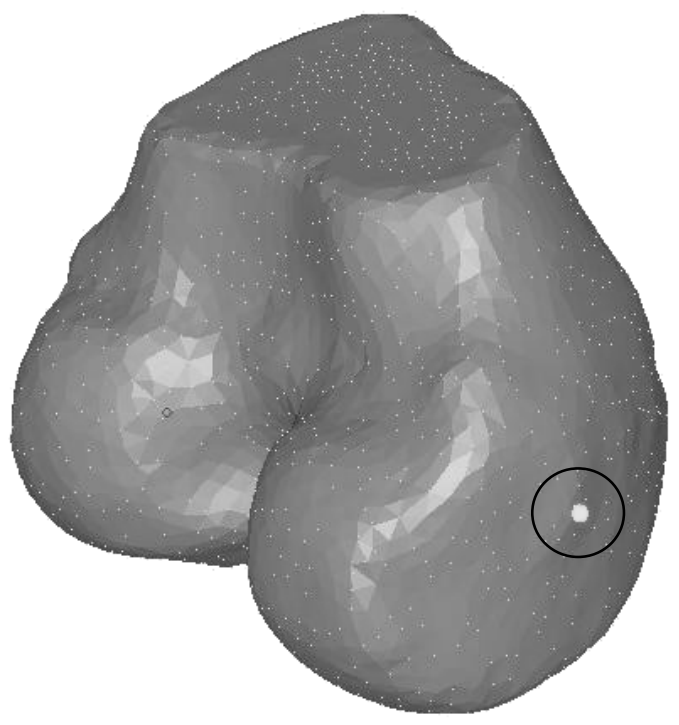

Figure 5: $\quad$ Example of FLE found with our routine.

To validate the routine developed, we decided to perform two different types of tests. The first test was performed to verify if the shape of the $3 \mathrm{D}$ models obtained corresponds with those of reference obtained using a spread commercial software (Mimics 11.02 and its MedCAD module (Materialise, Haasrode, Belgium). For this test we generated with our routine 5 different $3 \mathrm{D}$ models, 2 complete femur bones and 3 partial femurs. This test consisted exclusively of a visual comparison between the models, sufficient to check for any anomalies in the geometry of the models (fig. 6). 

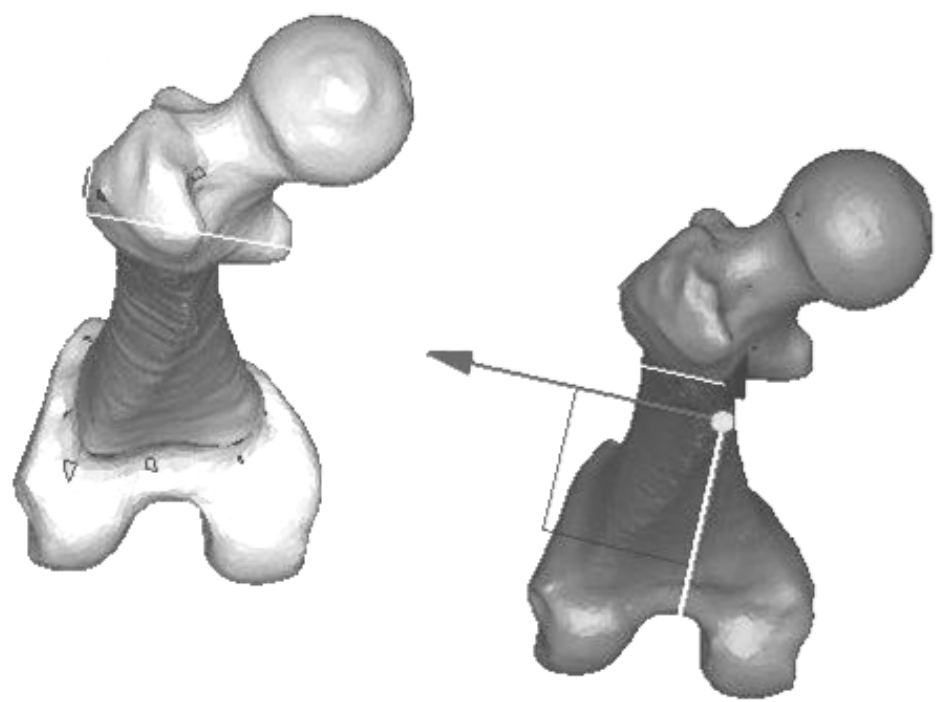

Figure 6: Comparison between the model obtained with our routine (1) and the one obtained with the spread commercial software of comparison (2).

The second test consisted of the generation of other 6 different 3D models of the femur on which 5 different users (only one expert in the localization of the anatomical landmarks) identified, for each model, the 3 anatomical landmarks 3 times each. We have obtained by each operator a set of 9 coordinates for each lower limb. Analyzing 6 lower limbs, we got a set of 54 coordinates for operator, being 5 the number of operators we got in total a set of 270 coordinates.

All these sets of coordinates have been used to estimate the intra-class correlation coefficient (ICC) and the inter-observer variability as the distance between the reference position of a landmark, obtained from the commercial software, to the observer position of the landmark [10]. The ICC describes how strongly units in the same group resemble each other. One prominent application is the assessment of consistency or reproducibility of quantitative measurements made by different observers measuring the same quantity. By definition, the ICC is evaluated according to the following formulation:

$$
\text { ICC }=\frac{\sigma_{b}^{2}}{\sigma^{2}}
$$

where the total variance of measurements by different observers is $\sigma^{2}$ on different subjects, and the variance between subjects is $\sigma_{\mathrm{b}}{ }^{2}$. ICC values range from 0 to 1 , indicating better agreement as the value approaches 1 . An ICC value higher than 0.75 should indicate excellent agreement. 


\section{Results}

As shown in table 1, the ICC values for all defined landmarks fall in a range between 0.996 and 0.999 , showing a good agreement between the observers and an excellent reliability for all the landmarks.

Table 1: $\quad$ ICC-Intra observer values and ICC-class values.

\begin{tabular}{|c|c|c|}
\hline Landmarks & ICC-IntraObserverValues & ICC-ClassValues \\
\hline Femoral Hip Center (FHC) & 0.9964 & 0.9961 \\
\hline Femoral Medial Epicondyle (FME) & 0.9999 & 0.9996 \\
\hline Femoral Lateral Epicondyle (FLE) & 0.9999 & 0.9996 \\
\hline
\end{tabular}

The inter observer variability for each landmark is shown in figures 7, 8, and 9 and the results are compared with the other obtained using the commercial software. The observed mean values and maximum values are displayed separately.

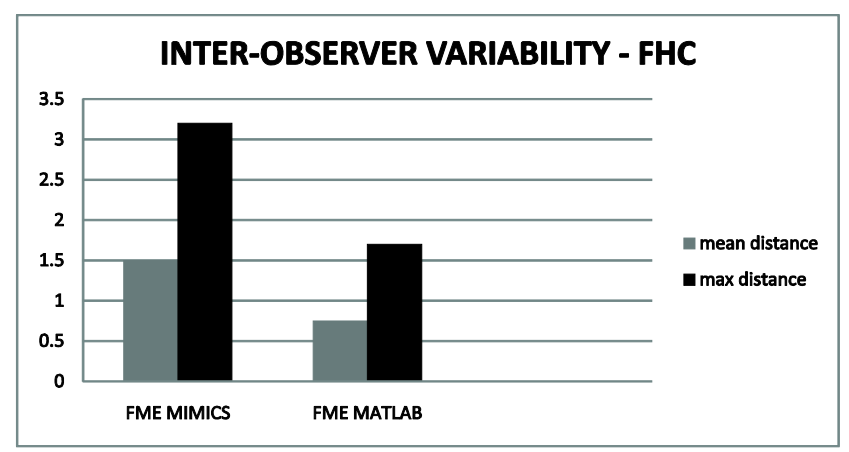

Figure 7: Comparison of the FHC inter-observer variability between the commercial software and our routine, shown as mean value and maximum value $[\mathrm{mm}]$.

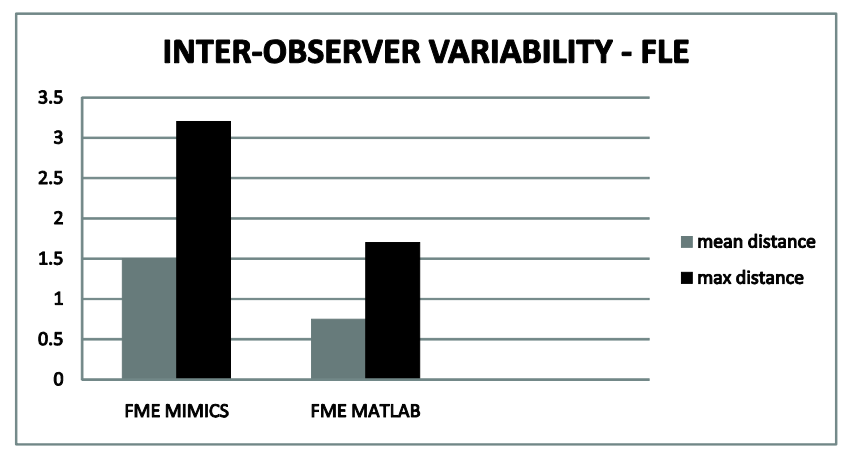

Figure 8: Comparison of the FLE inter-observer variability between the commercial software and our routine, shown as mean value and maximum value $[\mathrm{mm}]$. 


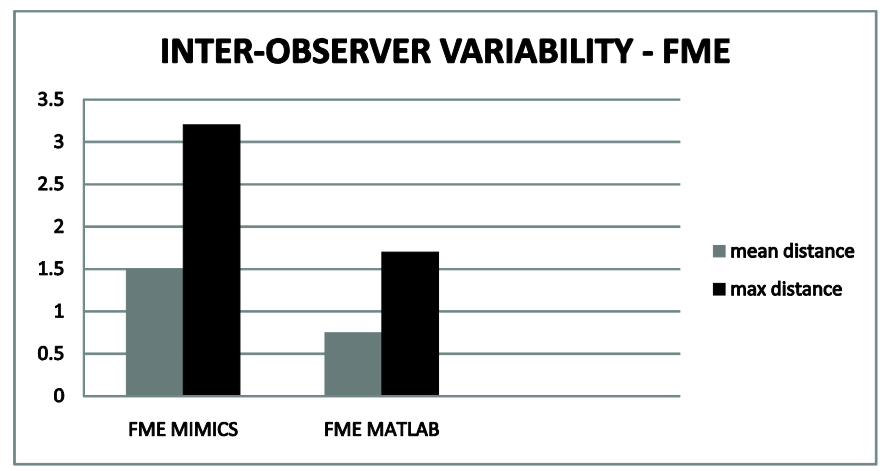

Figure 9: Comparison of the FME inter-observer variability between the commercial process and our routine, shown as mean value and maximum value $[\mathrm{mm}]$.

The average of the inter-observer variability obtained using our routine is $0.4 \mathrm{~mm}$ (range: $0.19 \mathrm{~mm}-0.6 \mathrm{~mm}$ ) and we can see, comparing this result with the one obtained using the commercial software, where the average is 1.9 (range: $0.3 \mathrm{~mm}-3.5 \mathrm{~mm}$ ), that the mean value is greatly improved moving from $1.9 \mathrm{~mm}$ to $0.4 \mathrm{~mm}$.

Maximum inter-observer variability is also improved moving from values between $0.8 \mathrm{~mm}$ to $8.6 \mathrm{~mm}$ for the commercial software to values between $0.38 \mathrm{~mm}$ to $1.8 \mathrm{~mm}$ for our routine.

Tables 2, 3 and 4, show the average and the standard deviation of the distance from observed position to the reference position that each operator $(\mathrm{Op})$ found for each landmark on all femur models.

Table 2: Average distance and standard deviation of the central femoral head.

\begin{tabular}{|c|c|c|c|c|c|c|}
\hline \multicolumn{7}{|c|}{ FEMORAL HIP CENTER - AVERAGE DISTANCE (mm) } \\
\hline $\begin{array}{c}\text { Average } \\
\text { distance }\end{array}$ & Femur1 & Femur2 & Femur3 & Femur4 & Femur5 & Femur6 \\
\hline Op.1 & 290.6 & 338.9 & 443.2 & 349.7 & 463 & 285.2 \\
\hline Op.2 & 289,9 & 337,9 & 443,5 & 349,3 & 462,3 & 285 \\
\hline Op.3 & 290 & 338 & 443,4 & 349,5 & 462,8 & 285,1 \\
\hline Op.4 & 290,2 & 337,6 & 443,6 & 349,7 & 462,5 & 285,2 \\
\hline Op.5 & 290, & 337,6 & 443,5 & 349,3 & 462,8 & 285,2 \\
\hline \multicolumn{7}{|c|}{ CENTRAL FEMORAL HEAD - STANDARD DEVIATION } \\
\hline $\begin{array}{c}\text { Standard } \\
\text { deviation }\end{array}$ & Femur1 & Femur2 & Femur3 & Femur4 & Femur5 & Femur6 \\
\hline Op.1 & 0 & 0 & 0 & 0 & 0 & 0 \\
\hline Op.2 & 0 & 0,04 & 0 & 0,3 & 0,1 & 0,04 \\
\hline Op.3 & 0,1 & 0,1 & 0,1 & 0,3 & 0,1 & 0,07 \\
\hline Op.4 & 0,1 & 0 & 0 & 0 & 0 & 0,07 \\
\hline Op.5 & 0 & 0 & 0 & 0 & 0 & 0 \\
\hline
\end{tabular}


Table 3: Average distance and standard deviation of the condylar lateral point.

\begin{tabular}{|c|c|c|c|c|c|c|}
\hline \multicolumn{7}{|c|}{ CONDYLAR LATERAL POINT - AVERAGE DISTANCE (mm) } \\
\hline $\begin{array}{c}\text { Average } \\
\text { distance }\end{array}$ & Femur1 & Femur2 & Femur3 & Femur4 & Femur5 & Femur6 \\
\hline Op.1 & 559,9 & 598,8 & 643,9 & 560,6 & 674 & 558,9 \\
\hline Op.2 & 560,5 & 598,8 & 642,4 & 559,8 & 673,4 & 558,9 \\
\hline Op.3 & 560,5 & 598,8 & 643,9 & 560,9 & 673 & 559,7 \\
\hline Op.4 & 557,6 & 600,4 & 643,9 & 560,9 & 673 & 559,7 \\
\hline Op.5 & 559,9 & 600,1 & 643,2 & 559,4 & 671,8 & 557 \\
\hline \multicolumn{7}{|c|}{ CONDYLAR LATERAL POINT - STANDARD DEVIATION } \\
\hline $\begin{array}{l}\text { Standard } \\
\text { deviation }\end{array}$ & Femur1 & Femur2 & Femur3 & Femur4 & Femur5 & Femur6 \\
\hline Op.1 & 0,5 & 0,4 & 0,4 & 0,4 & 0,2 & 0,2 \\
\hline Op.2 & 0 & 0,4 & 0 & 0 & 0,1 & 0,2 \\
\hline Op.3 & 0 & 0,4 & 0 & 0,4 & 0,5 & 0,01 \\
\hline Op.4 & 0,5 & 0,4 & 0 & 0,4 & 0,5 & 0,01 \\
\hline Op.5 & 0 & 0,4 & 2,3 & 0,2 & 0 & 0 \\
\hline
\end{tabular}

Table 4: Average distance and standard deviation of the condylar medial point.

\begin{tabular}{|c|c|c|c|c|c|c|}
\hline \multicolumn{7}{|c|}{ CONDYLAR MEDIAL POINT - AVERAGE DISTANCE (mm) } \\
\hline $\begin{array}{c}\text { Average } \\
\text { distance }\end{array}$ & Femur1 & Femur2 & Femur3 & Femur4 & Femur5 & Femur6 \\
\hline Op.1 & 520,9 & 604,9 & 688,8 & 595,5 & 631,9 & 523,8 \\
\hline Op.2 & 521,7 & 604,9 & 687,8 & 594,2 & 632,3 & 523,8 \\
\hline Op.3 & 519,6 & 604,9 & 688,8 & 594,5 & 631,5 & 523,6 \\
\hline Op.4 & 522,8 & 603,7 & 688,4 & 595,3 & 631,5 & 525,1 \\
\hline Op.5 & 523,4 & 604,2 & 688 & 594,3 & 632,1 & 529,6 \\
\hline \multicolumn{7}{|c|}{ CONDYLAR MEDIAL POINT - STANDARD DEVIATION } \\
\hline $\begin{array}{c}\text { Standard } \\
\text { deviation }\end{array}$ & Femur1 & Femur2 & Femur3 & Femur4 & Femur5 & Femur6 \\
\hline Op.1 & 2,1 & 0 & 0 & 0 & 0,5 & 0,4 \\
\hline Op.2 & 1,7 & 0 & 0,2 & 0,6 & 0,3 & 0,4 \\
\hline Op.3 & 0 & 0 & 0 & 0,1 & 0,2 & 0 \\
\hline Op.4 & 1 & 0,2 & 0,3 & 0,5 & 0,5 & 0,9 \\
\hline Op.5 & 0 & 0,7 & 0,3 & 0,2 & 0,5 & 0 \\
\hline
\end{tabular}

\section{Conclusions}

The aim of this study was to develop a semi-automatic, accurate, and reproducible routine able to generate $3 \mathrm{D}$ models and able to identify the position of anatomical landmarks on joint surfaces starting from a CT of a femoral bone.

From the results obtained, it is possible to see that there was a clear improvement in reproducibility and repeatability anatomical landmarks identification compared with the ones obtained with commercial software. The average of the inter-observer variability obtained using our routine is $0.4 \mathrm{~mm}$ (range: $0.19 \mathrm{~mm}-0.6 \mathrm{~mm}$ ) and we can see, comparing this result with the one obtained using the commercial software, where the average is 1.9 (range: $0.3 \mathrm{~mm}-3.5 \mathrm{~mm}$ ), that the mean value is greatly improved moving from $1.9 \mathrm{~mm}$ to $0.4 \mathrm{~mm}$. 
We can say that, using this developed and validated routine we are able to improve the quality of this landmarks identification.

For the moment this routine is able to work only with CT images, that is why we are already thinking to improve it for the MRI images in order to make our routine more complete and useful.

\section{References}

[1] Duncan, J.C. \& Ayache, N., Medical image analysis: progress over two decades and the challenges ahead. Journal IEEE Transactions on Pattern Analysis and Machine Intelligence, 22(1), pp. 85-106, 2000.

[2] Schafer R.C., Clinical Biomechanics - Musculoskeletal Actions and Reaction, Williams \& Wilkins: Baltimore, pp.711-717, 1991.

[3] Victor J., Van Doninck D., Labey L., Innocenti B., Parizel P.M. \& Bellemans J., How precise can bony landmarks be determined on CT scan of the knee. Knee, 16(5), pp. 358-365, 2009.

[4] http://www.mathworks.com/matlabcentral/

[5] Wong K.P., Medical image segmentation: methods and applications in functional imaging (Chapter 3). Handbook of Biomedical Image Analysis, Volume II: Segmentation Models Part B, ed.J.S. Suri, D.L. Wilson and S. Laxminarayan. SpringerLink, pp. 11-182, 2005.

[6] Dougherty G., Digital Image Processing for Medical Applications, Cambridge University Press, 2009.

[7] http://iso2mesh.sourceforge.net/cgi-bin/index.cgi

[8] Pérez-Pérez A., Alesan A. \& Roca L., Measurement error: inter- and intraobserver variability. an empiric study. International Journal of Anthropology, 5(2), pp. 129-135, 1990.

[9] LaPrade R.F., Ly T.V., Wentorf F.A. \& Engebretsen L., The posterolateral attachments of the knee: a qualitative and quantitative morphologic analysis of the fibular collateral ligament, popliteus tendon, popliteofibular ligament, and lateral gastrocnemius tendon. The American Journal of Sports Medicine, 31, pp.854-860, 2003.

[10] Bland J.M. \& Altman D.G., Measurement error. BMJ, 313, pp. 744-753, 1996. 